\title{
Some Observations on Human Control of a Bicycle
}

\author{
J. D. G. Kooijman and A. L. Schwab \\ Laboratory for Engineering Mechanics, \\ Delft University of Technology, \\ Mekelweg 2, 2628CD Delft, \\ The Netherlands \\ jodikooijman@gmail.com, a.l.schwab@tudelft.nl
}

Received: November 12, 2008.

\begin{abstract}
The purpose of this study is to identify human control actions in normal cycling. The task under study is the stabilization of the mostly unstable lateral motion of the bicycle-rider system. This is done by visual observation of the rider and measuring the vehicle motions. The observations show that no significant upper-body lean occurs and that most of the stabilizing control actions are done by steering control only. However, at very low forward speed a second control is added to the system: knee movement. Moreover, all control actions are performed at the pedalling frequency, whilst the amplitude of the steering motion increases rapidly with decreasing forward speed.
\end{abstract}

Keywords: Bicycle, Rider Control, Stabilization, Observations.

\section{INTRODUCTION}

Riding a bicycle is an acquired skill. At very low speed the bicycle is highly unstable. However, given some moderate speed the bicycle is easy to stabilize. These observations are confirmed by a stability analysis on a simple dynamical model of an uncontrolled bicycle [1] and some experiments [2]. Although there is little established knowledge on how we stabilize a bicycle, two basic features are known: some uncontrolled bicycles can balance themselves given some initial speed, and one can balance a forward moving bicycle by turning the front wheel in the direction of the undesired lean. Still, observing a rider on a bicycle, not only the steer is moved but also the upper body and other extremities are moving. These rider body motions are even more profound in riding a motorcycle [3].

The purpose of this study is to identify the major human control actions in normal bicycling where we focus on the stabilizing task only, no tracking. The identification is done by visual observation of the rider and measurement of the vehicle motions on an instrumented bicycle, see Figure 1. In order to observe the human control actions a number of experiments were carried. First a typical town ride was made to investigate what sort of actions take place during normal riding. After this, experiments were carried out in a controlled environment on a large treadmill ( $3 \times 5 \mathrm{~m}$ ) at various speeds. One and the same bicycle was used during all the experiments. The bicycle was ridden by two averagely skilled riders. Three cases were considered; normal riding, towing and normal cycling with lateral perturbations. This, to identify the effect upper body motion and the effect of the pedalling motion on the control. The rider was told to simply stabilize the bicycle and to generally ride in the longitudinal direction of the 
treadmill; no tracking task was set. Recorded data were the rigid body motions of the bicycle rear frame and the front assembly. The rider motion relative to the rear frame was recorded on video.

\section{INSTRUMENTED BICYCLE}

A common, standard Dutch bicycle, the Batavus Browser (Model 2008) was chosen for the experiments and is shown in Figure 1. This is a bicycle of conventional design, fitted with a 3-speed SRAM rear hub and coaster brakes. Some of the peripheral components were removed in order to be able to install measurement equipment.

The bicycle was equipped with a bullet-style camera with $2.8 \mathrm{~mm}$ (wide angle) lens at the front. The camera was directed towards the rider and rotated 90 degrees clockwise to get portrait aspect ratio. The video signal was recorded, via the AV-in port, on DV tape of a Sony Handycam located on the rear rack of the bicycle. The bullet camera was placed horizontally, approximately $65 \mathrm{~cm}$ in front of the handlebars and $1.2 \mathrm{~m}$ above the ground and held in place by a carbon-fiber boom connected to the down-tube of the rear frame.

A National Instruments' CompactRIO (type 9014) computer was used for data collection. The CompactRIO was installed on the rear rack of the bicycle and was fitted with a 32 port-analogue in slot and a 4 port analogue-out slot as well as a wireless modem for a wireless connection with a "ground station" router, to which a laptop was connected. The measurement system is able to run autonomously once a measurement sequence is initiated. The CompactRIO was powered by a $11.1 \mathrm{~V}$, 1500mAh Lithium Polymer battery which was also placed on the bicycle's rear rack.

The recorded signals were the lean, yaw and steering rates, the steering angle, the rear wheel speed and the pedalling cadence frequency. The angular rates were measured using 3 Silicon Sensing CRS03, single axis angular rate sensors with a rate range of $+-100 \mathrm{deg} / \mathrm{s}$. The steering angle was measured using a potentiometer placed on the rear-frame against the front of the head tube and connected via a belt and pulley pair. The angular rate sensors and the angular potentiometer were powered by a $4.8 \mathrm{~V}$, 2100mAh Nickel Cadmium battery. The forward speed was measured by measuring the output voltage of a Maxon motor that was driven by the rear wheel. The Cadence frequency was measured by a reed-relay placed on the rear frame, and a magnet placed on the frame side of the crank-arm. 


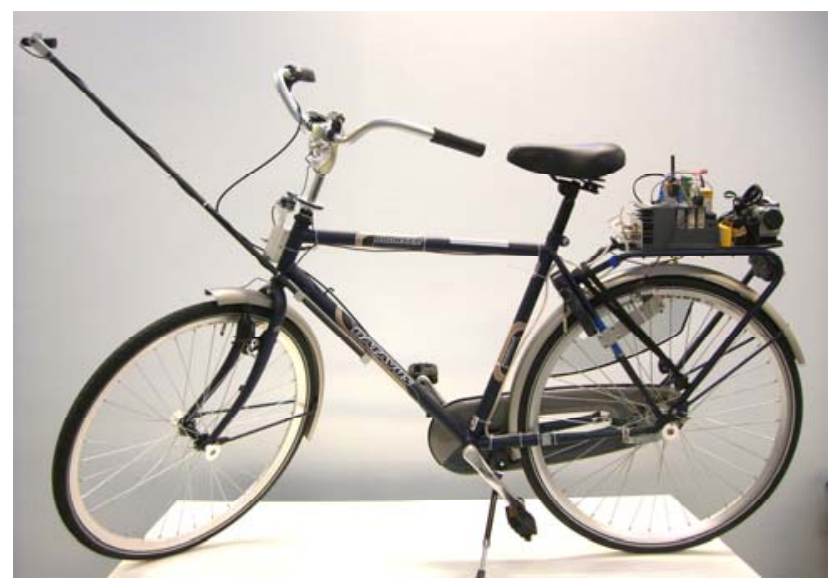

Fig. 1 The instrumented bicycle with camera boom and video camera lens at the front. On the rear rack the measurement computer (NI CompactRIO), video camcorder and battery packs are positioned. Measured signals are the steering angle (potentiometer), steering-rate, rear-frame lean- and yaw-rate and forward speed

The uncontrolled dynamics of the bicycle rider combination can be described by the linearized stability of a model of the bicycle [1]. This model consists of four rigid bodies, viz. the rear frame with rigid rider connected, the front frame steering assembly, and the two wheels, connected by ideal hinges and where the wheel have idealized pure-rolling contact with level ground (no tires models). For the instrumented bicycle and one of the riders this linearized stability is depicted in Figure 2. At low speed the important motion is the unstable oscillatory weave motion. This weave motion becomes stable around $23 \mathrm{~km} / \mathrm{h}$, the so-called weave speed. At higher speeds the non-oscillatory capsize motion becomes mildly unstable but since this instability is so mild it is not really important. Summarizing: the instrumented bicycle rider combination is in need of human stabilizing control below $23 \mathrm{~km} / \mathrm{h}$ and is selfstable above.

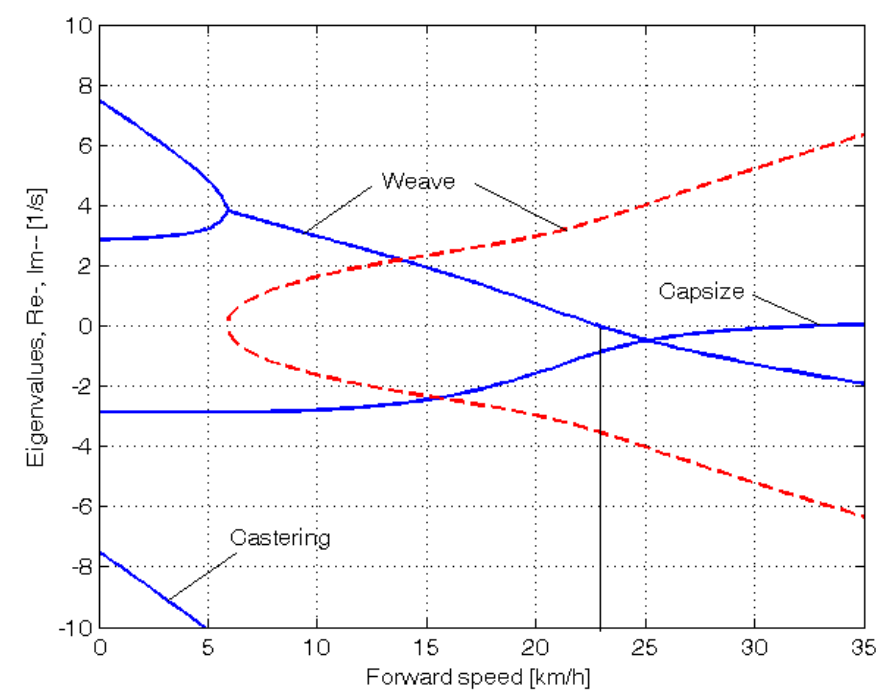

Fig. 2 Eigenvalues for the linearized stability analysis on an uncontrolled bicycle-rider combination for the steady upright motion in the forward speed range of 0-35 km/h. Solid lines are real parts, dotted lines are imaginary parts. The bicycle is practically self-stable from the weave speed, $23 \mathrm{~km} / \mathrm{h}$, and above. 


\section{TOWN RIDE EXPERIMENT}

As a first step in human rider control observations a short, 15 minute, ride around town was carried out. This experiment took place under normal riding circumstances (dry weather, daylight, etc.), on roads that the rider was familiar with. The course covered included a round-a-bout, dedicated cycling paths, speed-bumps, pavement, normal tarmac roads, tight bends in a residential area and the rider had to stop at a number of traffic lights. There were no special precautions taken and the experiment was carried out amongst other traffic. Initially this experiment was only intended for testing the measurement equipment however upon inspecting, from the recorded video material and measured data two observations could be made:

1. from the video material it was immediately clear that very little upper body lean relative to the rear-frame, was carried out during the whole ride. The relative upper body lean that was noted appeared to be as a result of pedalling. Only in the last few seconds prior to a sharp corner was an upper body lean angle observed - indicating that the lean was probably carried out for tracking reasons.

2. from the recorded data, part of which is shown in Figure 3, it was noted that most of the time only very small steering actions are carried out (note that the potentiometer was not aligned with the steering axis giving the -5 degree offset). Only when the forward speed drops, prior to making a corner, are large steering angles (> $15 \mathrm{deg}$ ) seen.
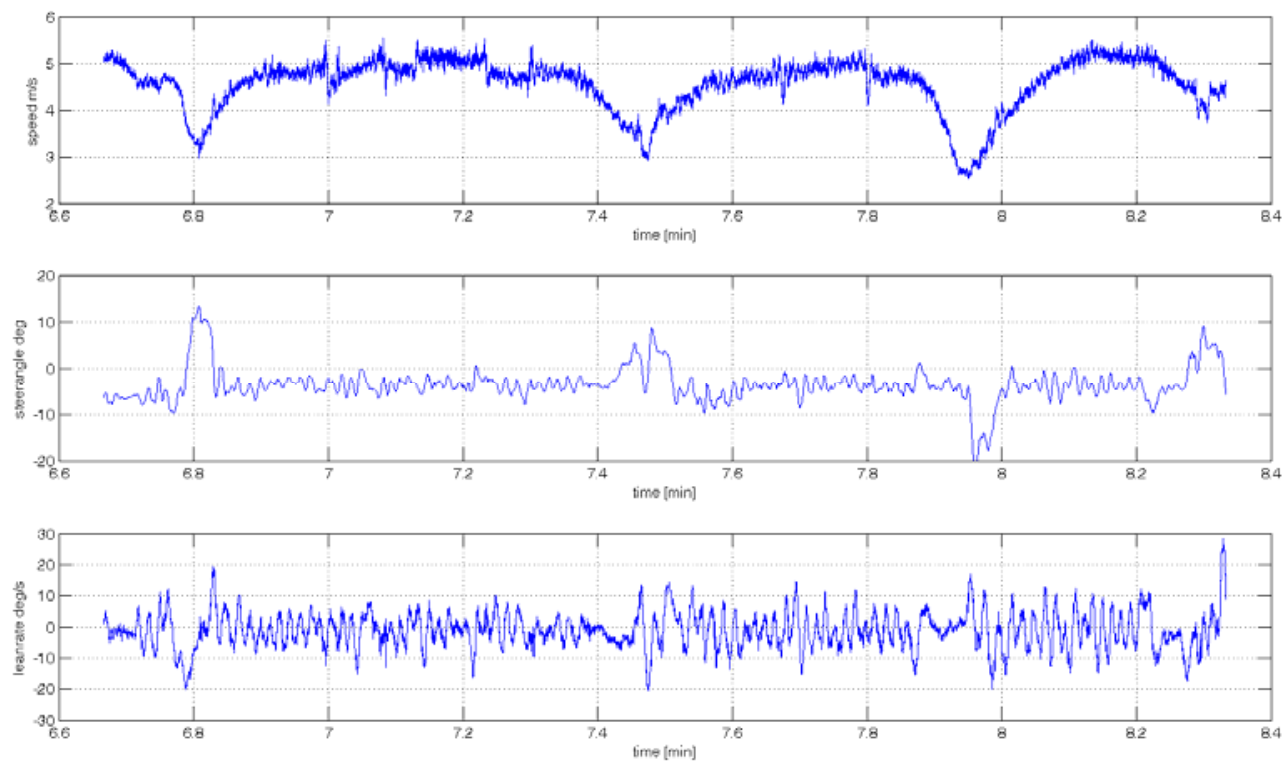

Fig. 2. Part of the data collected during the town ride. Shown are the bicycle forward speed (top), steering angle (middle) and rear-frame lean rate (bottom) versus time. 


\section{TREADMILL EXPERIMENTS}

The town ride showed to many distracting external disturbances. Therefore, a more controlled environment was sought to study human rider control for stabilizing tasks. A large treadmill, shown in Figure 3, of the faculty of Human Movement Sciences at the VU Amsterdam was used. This eliminated road unevenness, side wind, traffic and other disturbances.

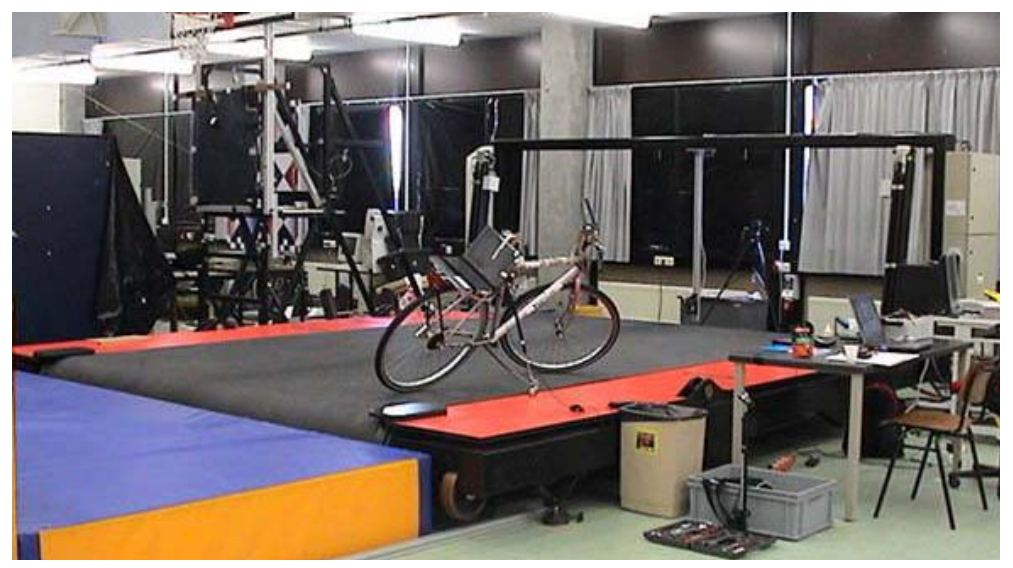

Fig. 3. Large treadmill, $3 \times 5 \mathrm{~m}$, max speed $35 \mathrm{~km} / \mathrm{h}$, courtesy of the Faculty of Human Movement Sciences, VU Amsterdam.

The experiments were carried out by two, male, average ability, riders of different build on the same bicycle. The saddle height was adjusted for each rider to ensure proper seating and cycling.
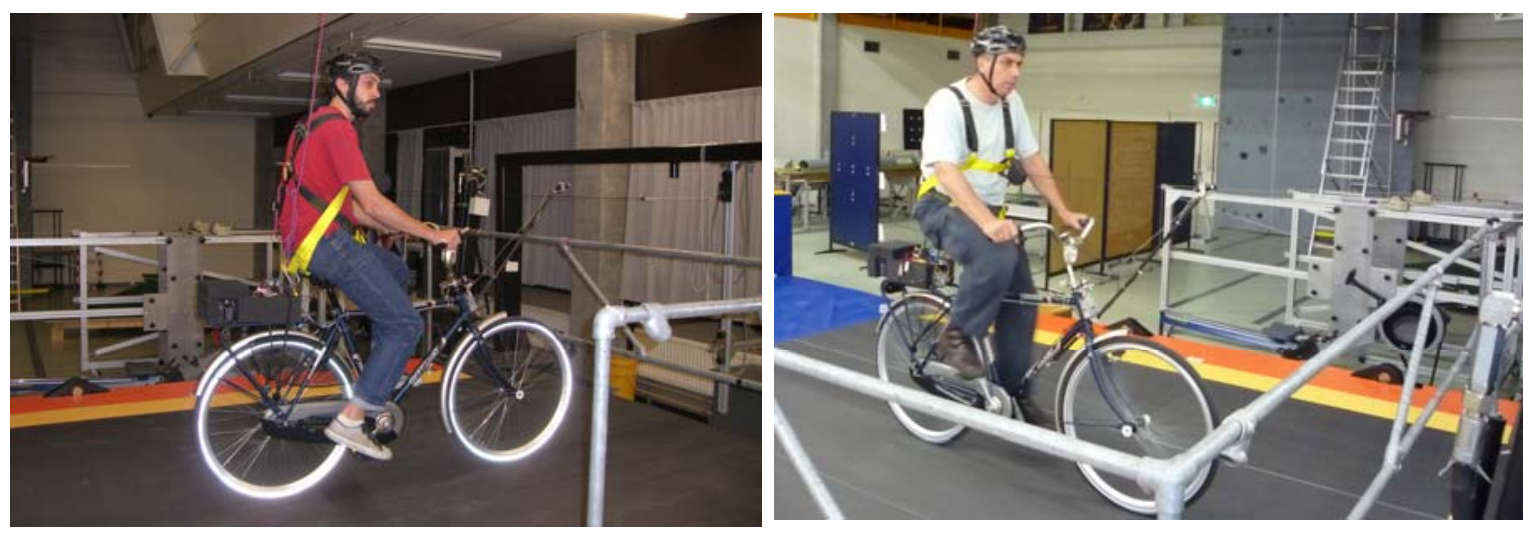

Fig. 4 The two average riders riding the instrumented measurement bicycle on the treadmill

Three experiments were carried out; normal cycling, towing and cycling with lateral perturbations. Each experiment was carried out at 6 different speeds: 30, 25, 20, 15, 10 and $5 \mathrm{~km} / \mathrm{h}$. So in total 36 experiments were carried out. The riders were instructed to stay on the treadmill and to generally ride in the longitudinal direction of the treadmill but not to concentrate on their position on the treadmill in order to eliminate the 
tracking task. Data was collected for 1 minute during each experiment with a $100 \mathrm{~Hz}$ sample rate. Video footage can be found at the website [4].

\subsection{Normal Cycling; Pedalling}

During this experiment the rider was pedalling normally. At the 2 highest speeds (30 and $25 \mathrm{~km} / \mathrm{h}$ ) the third gear was used, at 20 and $15 \mathrm{~km} / \mathrm{h}$ the second gear was used and during the tests at 10 and $5 \mathrm{~km} / \mathrm{h}$ the first gear was used.

From the visual inspection of the video footage it was immediately clear that no lean action was taking place during cycling other than that resulting directly from the pedalling motion. Furthermore it was noted that during the low speed, $5 \mathrm{~km} / \mathrm{h}$, the riders' upper body was almost stationary (i.e. rigidly attached to the rear-frame). However the riders' knees showed significant lateral motion in order to stabilize the bicycle at this speed.

The third observation was that the rider was turning the handle-bars far more at lower speeds than at higher speeds. This observation was confirmed by the measured steering angle data. In Figure 5 the last 30 seconds of the steering angle measurements for $30 \mathrm{~km} / \mathrm{h}, 15 \mathrm{~km} / \mathrm{h}$ and $5 \mathrm{~km} / \mathrm{h}$ are shown for the second rider. The figure clearly shows that the steering angle increases by a factor of 10 when the speed drops from 30 $\mathrm{km} / \mathrm{h}$ to $5 \mathrm{~km} / \mathrm{h}$.

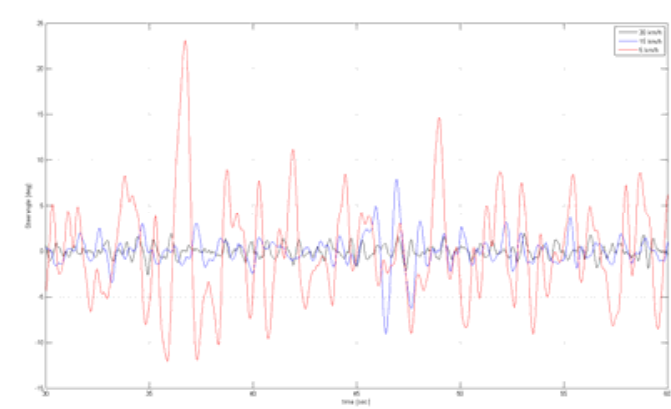

Fig. 5 The time history plot of the steering angle for normal cycling at $30 \mathrm{~km} / \mathrm{h}, 15$ $\mathrm{km} / \mathrm{h}, 5 \mathrm{~km} / \mathrm{h}$.

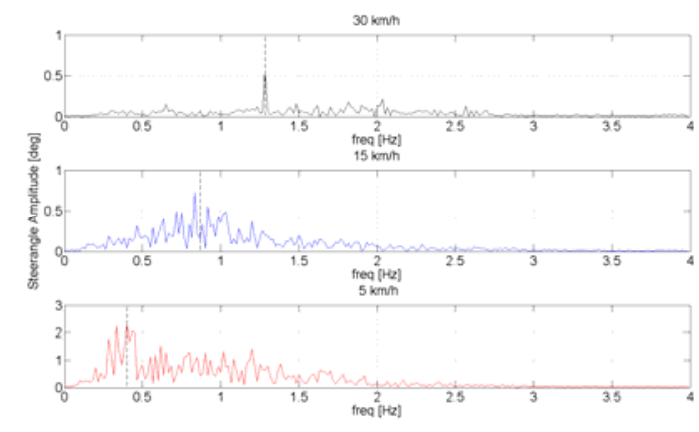

Fig. 6 The frequency plot of the steering angle for normal cycling at $30 \mathrm{~km} / \mathrm{h}, 15$ $\mathrm{km} / \mathrm{h}, 5 \mathrm{~km} / \mathrm{h}$.

The frequency content of the steering signal was investigated to determine at which frequency the rider turns the handlebars. In Figure 6 the results are shown for the 30 $\mathrm{km} / \mathrm{h}, 15 \mathrm{~km} / \mathrm{h}$ and $5 \mathrm{~km} / \mathrm{h}$ experiments. The vertical dashed line in each of the plots indicates the measured pedalling frequency. From the figure it is clear that during normal pedalling most of steering action takes place at or around the pedalling frequency irrespective of the speed that the bicycle is moving.

\subsection{Towing; No Pedalling}

Since pedalling showed such a dominant factor in the previous experiments a number of non-pedalling experiments where conducted. The bicycle and rider were 
towed during this experiment by a rope connected to the bicycle rear-frame at the lower end of the head tube, while the rider kept the pedals in the horizontal position.

The video footage once again revealed that there was no upper body leaning at any of the measured speeds. At $5 \mathrm{~km} / \mathrm{h}$ there was some lateral knee motion but it was not as significant as during the normal pedalling at the same speed. The measured steer angle plot shown in Figure 7 shows that the steering angle at $5 \mathrm{~km} / \mathrm{h}$ is smaller than for the case with pedalling, Figure 5. The amplitude of the steering angle at $15 \mathrm{~km} / \mathrm{h}$ and $30 \mathrm{~km} / \mathrm{h}$ however are very similar to those seen with pedalling.

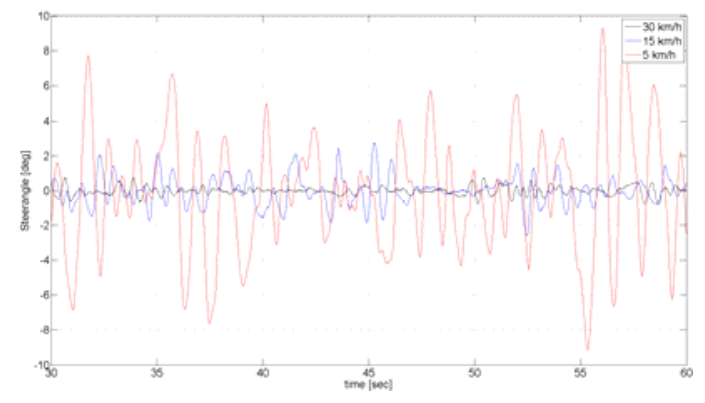

Fig. 7 The time history plot of the steering angle for towing at $30 \mathrm{~km} / \mathrm{h}, 15$ $\mathrm{km} / \mathrm{h}, 5 \mathrm{~km} / \mathrm{h}$.

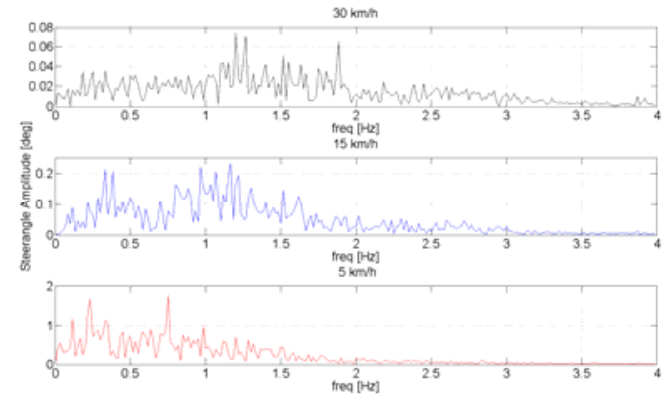

Fig. 8 The frequency plot of the steering angle for towing at $30 \mathrm{~km} / \mathrm{h}, 15 \mathrm{~km} / \mathrm{h}, 5$ $\mathrm{km} / \mathrm{h}$.

The frequency plot for the towing experiment, Figure 8, shows steering control in a broad frequency band. The expected peaks at the weave frequencies corresponding to the forward speeds does not occur.

\subsection{Perturbing; Pedalling}

Next, we were interested in how the human rider recovers from a sudden lateral impulse. During this experiment, similar to the normal riding experiment, the rider was pedalling normally and used the same gears at the different speeds as during the normal cycling experiment. The bicycle was perturbed by applying a lateral impulse to the rear frame. The impulse was applied by a manually actuated rope tied to the seat tube. The rider could not see the rope being actuated to ensure that he was unprepared.

The video footage showed that as a result of the lateral perturbation the bicycle was pulled away from under the rider causing a short transient lean motion. The upper body lagged behind the lower body and bicycle. However, during the following recovery of the bicycle to the upright, straight ahead position, no body lean could be noted other than that as a result of the normal pedalling.

A second phenomenon that was observed was that at all speeds there was lateral knee motion during the short transient recovery process of the bicycle to the upright position. This lateral knee motion was very large during the low speed measurement and much smaller at the high speed, but even at the high speed it was visible.

The angle that the handlebars were turned during/after a perturbation once again increased with decreasing speed. This can be seen in the time series from Figure 9 . The figure also shows that the time required to recover the bicycle to the upright position increases with decreasing speed. 


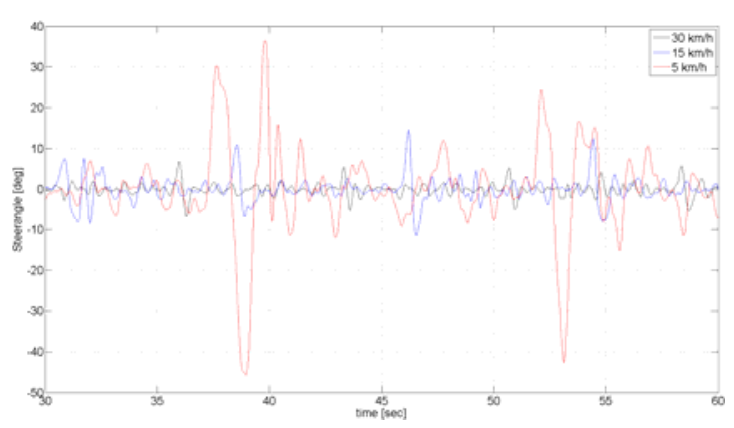

Fig. 9 The time history plot of the steering angle for perturbed cycling at $30 \mathrm{~km} / \mathrm{h}, 15 \mathrm{~km} / \mathrm{h}, 5 \mathrm{~km} / \mathrm{h}$.

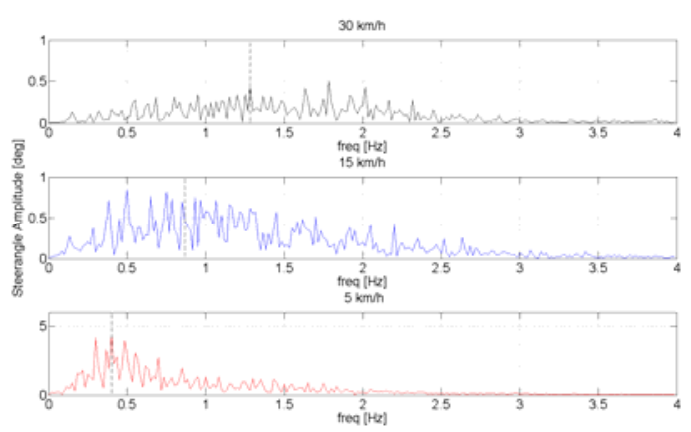

Fig. 10 The frequency plot of the steering angle for perturbed cycling at $30 \mathrm{~km} / \mathrm{h}, 15$ $\mathrm{km} / \mathrm{h}, 5 \mathrm{~km} / \mathrm{h}$.

Figure 10 shows the frequency spectrum for the measured steering signals. Once again, for all speeds, most of the steer control action appears to be around the pedalling frequency.

\section{CONCLUDING REMARKS}

The observations show that human stabilizing control for the lateral motions of a bicycle during normal cycling does not show any significant upper body lean, and that most of the stabilizing control actions are done by steering control. Only, at very low forward speed a second control is added to the system: knee movement. Moreover, all control actions are mainly performed at the pedalling frequency whilst the amplitude of the steering motion increases rapidly with decreasing forward speed.

Acknowledgements - We like to thank Jason Moore for his contribution to the instrumented bicycle, his enduring enthusiasm in the project, and his test-rider performances, and Knoek van Soest for the usage of the treadmill at VU Amsterdam.

\section{REFERENCES}

[1] Meijaard, J. P. - Papadopoulos, Jim M.- Ruina, Andy - Schwab, A. L.: Linearized dynamics equations for the balance and steer of a bicycle: a benchmark and review. Proceedings of the Royal Society A, 2007. 463, (p. 1955-1982).

[2] Kooijman, J. D. G. - Schwab, A. L. - Meijaard, J. P.: Experimental validation of a model of an uncontrolled bicycle, Multibody System Dynamics, 2008. 19(1-2), (p. 115-132).

[3] Cossalter, V. - Motorcycle Dynamics, Race Dynamics, 2002, Greendale, WI.

[4] Video footage at: http://www.tam.cornell.edu/ als93/Bicycle/index.htm\#Oct28 